Original Research Paper

\title{
MDS Algorithm for Encryption
}

\author{
${ }^{1}$ Mani Arora, ${ }^{2}$ Derick Engles and ${ }^{3}$ Sandeep Sharma \\ ${ }^{1}$ Khalsa College, Amritsar, Punjab, India \\ ${ }^{2,3}$ Guru Nanak Dev University, Amritsar, Punjab, India
}

Article history

Received: 3-08-2013

Revised: 6-01-2015

Accepted: 13-03-2015

Corresponding Author:

Mani Arora

Khalsa College, Amritsar,

Punjab, India

Email:mani_mcain@yahoo.com

\section{Introduction}

In the digital world with the widespread use of information technologies and the rise of digital computer networks in many areas of the world, securing the exchange of information has become a crucial task (McConnell, 2002). Cryptography is the methods that allow information to be sent in a secure form in such a way that the only receiver able to retrieve this information. The era of modern cryptology is generally agreed to have started in 1949, when Shannon transformed cryptography from an art to a science with the publication of a paper entitled "Communication theory of secrecy systems" (Diffie and Hellman, 1976; 1979). However, while cryptology took a new fundamental direction from that point on, most of the major innovations in the field date from the last 30 years. Modern cryptography involves the use of keys for data signing, encoding and decoding. Some keys are distributed privately and some publicly. Level of protection is varied for every situation and depends on the encryption technique used for coding. One way to estimate techniques on this level is to estimate how much CPU time would be required on a machine of a given processing speed to iterate through all possible keys to the encoded data based upon the permutation and second is how much secure the data is while transmitting. The basic goal of our work is along with these two measures cryptography algorithm must also be efficient in reducing the size of encrypted text referred as cipher text. In our earlier paper (Mani and Derick, 2010) we have used the term reduced size cipher text to convert plain text to cipher text. In this study we are modifying the cryptographic technique to provide better results.

\section{Proposed Technique}

The proposed technique intends to encrypt the plain text with a prechosen mathematical function along with the objective of reduced size cipher text. During encryption and decryption process, two dictionaries are referred primary dictionary as shown in Table 1 and secondary dictionary as shown in Table 2. Dictionary technique is used as it provide compression efficiency as well as fast decompression mechanism. The basic idea is to take the advantage of commonly occurring instruction sequences by using a dictionary. The repeating occurrences are replaced by a codeword. Primary dictionary will be static in nature while secondary dictionary will be dynamic. It depends on the nature of text being encoded. The online building of the secondary dictionary in the primary memory ensures the single pass over the data and the dictionary need to be transmitted over the network. In this previous technique we not focused on frequent occurrence of alphanumeric and numeric data. Since any digit took 2 bytes i.e., 16 bits in memory but if we provide code of 12 bits to every digit, it will reduce the size of cipher text as well. So the modified primary dictionary will contain codes for words, numeric data and alphanumeric data. We have still focused on English language only while creating dictionaries.

\section{Primary Dictionary}

The dictionary contains words and numeric data which are probably most frequently used along with the codes* (which will be explained later). The dictionary will be fixed in size and the codes too. Even if someone cracks the dictionary and codes, still our technique is going to work because it is based on both primary and secondary dictionary and secondary dictionary is not fixed. 
Table 1 . Primary dictionary

\begin{tabular}{|c|c|}
\hline Code & String \\
\hline 000000000001 & 0 \\
\hline 000000000010 & 1 \\
\hline 000000000011 & 2 \\
\hline 000000000100 & 3 \\
\hline 000000000101 & 4 \\
\hline 000000000110 & 5 \\
\hline 000000000111 & 6 \\
\hline 000000001000 & 7 \\
\hline 000000001001 & 8 \\
\hline 000000001010 & 9 \\
\hline 000000001011 & after \\
\hline 000000001100 & off \\
\hline 000000001101 & I \\
\hline 000000001110 & or \\
\hline 000000001111 & an \\
\hline 000000010000 & $\mathrm{a}$ \\
\hline 000000010001 & as \\
\hline 000000010010 & in \\
\hline 000000010011 & ok \\
\hline 000000010100 & It \\
\hline 000000010101 & is \\
\hline 000000010110 & on \\
\hline 000000010111 & at \\
\hline 000000011000 & of \\
\hline 000000011001 & If \\
\hline 000000011010 & we \\
\hline 000000011011 & my \\
\hline 000000011100 & do \\
\hline 000000011101 & am \\
\hline 000000011110 & $\mathrm{pm}$ \\
\hline 000000011111 & be \\
\hline 000000010000 & to \\
\hline 000000100001 & by \\
\hline 000000010010 & can \\
\hline 000000010011 & the \\
\hline 000000010100 & was \\
\hline 000000010101 & sat \\
\hline 000000010110 & for \\
\hline 000000010111 & not \\
\hline 000000011000 & has \\
\hline 000000011001 & had \\
\hline 000000011010 & him \\
\hline 000000011011 & her \\
\hline 000000011100 & other \\
\hline 000000011101 & which \\
\hline 000000011110 & where \\
\hline 000000011111 & you \\
\hline 000000110000 & your \\
\hline 000000110001 & some \\
\hline 000000110010 & too \\
\hline 000000110011 & who \\
\hline 000000110100 & Its \\
\hline 000000110101 & and \\
\hline 000000110110 & whatever \\
\hline 000000110111 & herself \\
\hline 000000111000 & bar \\
\hline 000000111001 & can't \\
\hline 000000111010 & don't \\
\hline 000000111011 & there \\
\hline 000000111100 & does \\
\hline 000000111101 & into \\
\hline
\end{tabular}

Table 1. Continue 000000111110 000000111111 000001000000 000001000001 000001000010 000001000011 000001000100 000001000101 000001000110 000001000111

000001001000

000001001001

000001001010

000001001011

000001001100

000001001101

000001001110

000001001111

000001010000

000001010001

000001010010

000001010011

000001010100

000001010101

000001010110

000001010111

000001011000

000001011001

000001011010

000001011011

000001011100

000001011101

000001011110

000001011111 this

back

were

four

that

back

from

form

most

word

whom

able

here

must

did

didn't

like

national

Table 2. Secondary dictionary

000100000000

000100000001

000100000010

000100000011

000100000100

000100000101

000100000110

000100000111

000100001000

000100001001

000100001010

000100001011

000100001100

000100001101

000100001110

000100001111

000100010000

000100010001

000100010010

000100010011

000100010100

000100010101

000100010110

000100010111

000100011000 
Table 3. Contents of key file 111100001111

To maintain this dictionary plain text file is used and for processing we read the file into memory as associative arrays. Associative arrays maps arbitrarily typed objects to arbitrarily typed objects. Data structures used to represent associative array when initialized in memory will be linked list. For searching a word in the dictionary, simple linear search is used. We are using 12 bit codes so total 4096 words can be stored in this dictionary.

\section{Secondary Dictionary}

The dictionary is not fixed. This dictionary will be empty when initialized. Every time the algorithm come across the string in pass2, it will add it to dictionary and assign a code to it. Starting code for first string in secondary dictionary will be fixed. The next codes can be obtained by doing increment of one step. As this dictionary will be created during runtime so it's difficult to crack the encryption. This dictionary will also be stored in same file that for primary dictionary is used.

\section{Encryption Algorithm}

The algorithm will start with initializing a primary dictionary and variable $\mathrm{S}$, which will be initially empty. We read the file containing dictionary into memory in linked list structure. The variable $\mathrm{S}$ will read plain text data file word by word and comparisons in dictionary will be made with help of this variable. In the algorithm each word of plain text will be first searched in primary dictionary, this is done using linear search. If the word is present in primary dictionary then it will be replaced by corresponding code assigned to it and stored in encoded output file. Codes are in binary as binary take less memory space than any other data type. Codes are fixed for each data word so it can't be changed later on. Size of code allocated to each string will be 12 bits that is any dictionary can contain maximum 4096 entries. In this modified version of the previous algorithm we are considering first 10 entries of the numeric data in primary dictionary. As we know otherwise numeric digit took 2 byte (16 bits) space in memory but here we are allocating 12 bits fixed code for every numeric digit which will also compress the data and finally the cipher text. Also some codes will be allocated to common alphanumeric data so that data can be compressed.

If the word is not found in primary dictionary then it is searched in secondary dictionary. If the word is present in secondary dictionary its corresponding code will be substituted to encoded output file, if not present then that word is substituted in secondary dictionary and the new code is generated to it by incrementing the code by 1 of last entry in secondary dictionary. The code for first entry in secondary dictionary will be fixed and rest codes will be obtained by incrementing each code by 1.Secondary dictionary will be of variable size.

After these pass we will get encoded output file. Further in the algorithm we use a predetermined mathematical function XOR to further encrypt the encoded output. As code assigned to each word is of 12 bits, so in pass 3 each 12 bit block will be XOR with a secret key to get final output which will be a cipher text in reduced size then plain text.

The above procedure is also explained with the help of flowchart i.e., Fig. 1.

\section{The Algorithm for Proposed Technique is:}

1. Initialize $\mathrm{S}$ as an empty string

2. Read primary dictionary in memory

3. Do while NOT EOF

Read the next word from the file

If this word is in primary dictionary get corresponding code from primary dictionary

else

Write the code in the output file

Read secondary dictionary in file

If this word is in secondary dictionary see the corresponding code from secondary dictionary

Write the code in the output file

else

add this word to secondary dictionary assign the next code obtained by incrementing previous code by 1 used to substitute the new code in the output file

4. Read the output file

5. Do while NOT EOF

Read key from file in memory

Read next 12 -bit block from file

Perform XOR operation between key and 12-bit block

Write the code in final output file

6. End

\section{Primary Dictionary}

Primary dictionary is fixed so sender and receiver both before transmission of cipher text will know it.

\section{Algorithm to Create Primary Dictionary}

1. Start with prechosen code and assign first word to it

2. Add new word to dictionary

3. Assign code to new word by incrementing previous code by 1

\section{Secondary Dictionary}

Secondary dictionary is dynamic in nature. It will be created at runtime.

\section{Algorithm to Create Secondary Dictionary}

1. Initialize $A$ as an empty string

2. Do till EOF

Read the file till any string is

encountered

Assign string to A

Add this string to dictionary

3. Start with prechosen code and assign first string to it

4. Assign code to new word by incrementing previous code by 1 


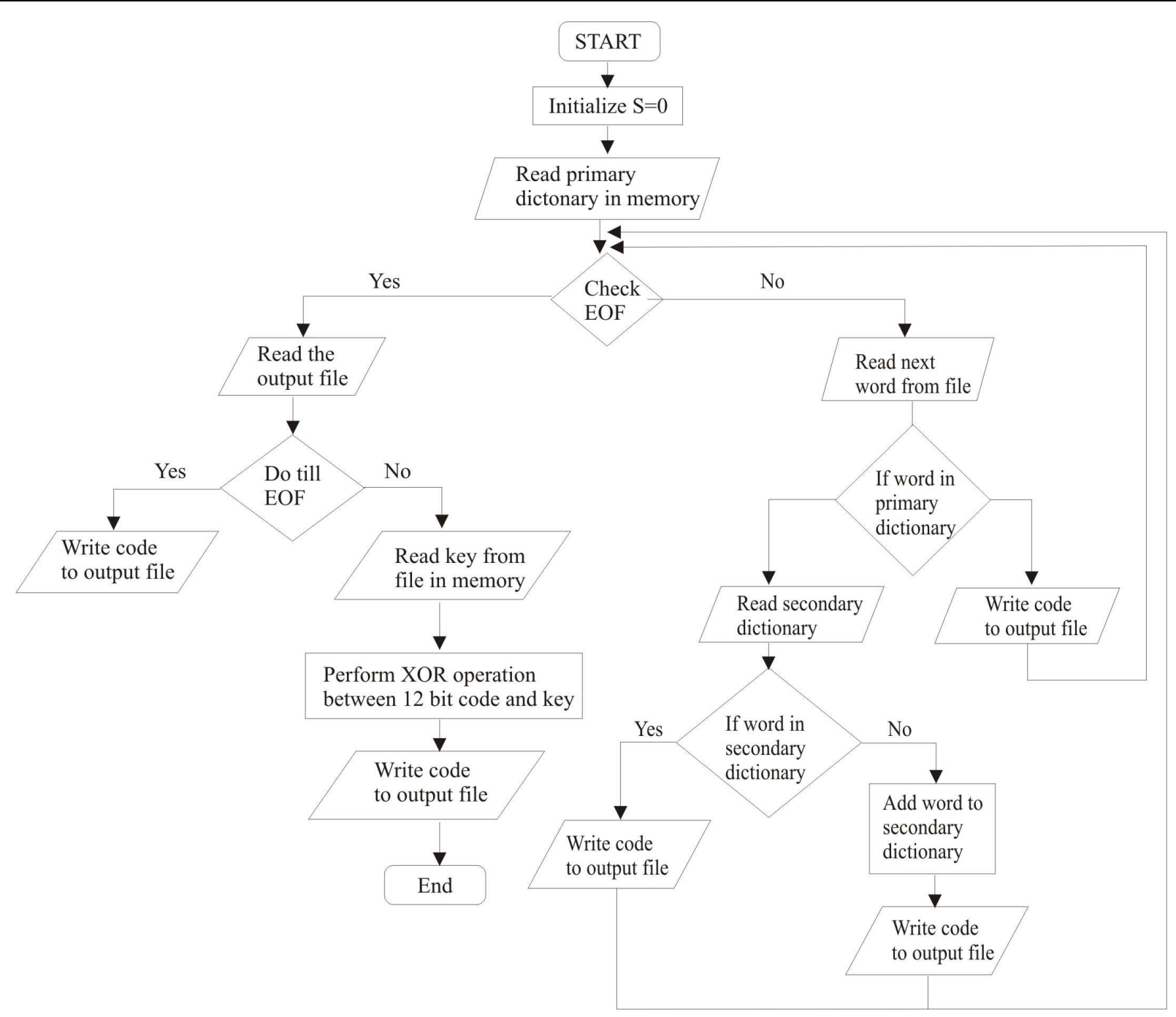

Fig.1. Flowchart

\section{Flowchart for the Algorithm is as Follows}

\section{Example}

For Example the plain text given below is encrypted after pass 1 and pass 2 as shown below.

\section{Plain Text that is to be Encrypted is:}

\section{Confidentiality}

The information cannot be understood by anyone for whom it was unintended. Confidentially is the protection of transmitted data from passive attacks. The other aspect of confidentiality is the protection of traffic flow from analysis. This requires that an attacker not be able to observe the source and destination frequency length or other characteristics of the traffic on a communication facility.
Pass1: In this pass primary dictionary given as Table 1 and secondary dictionary given as Table 2 is referred for substituting codes in place of text:

0000000000100000010101100001000000000000 0101010100000001001100010000000100000001 0010000000011111000000010011000000100001 0000010000000001011000000100100000000001 0100000000010100000100000101000001010000 0001000000000000000101010000000100110001 0000011000000001100000010000011100010000 1000000001000100000100001001000100001010 0000010100000000000100110000000111000001 0000101100000001100000010000000000000001 0101000000010011000100000110000000011000 0001000011000001000011010000010001000001 0000111000000101000000000011111000010000 1111000001000010000000001111000100010000 
0000000101110000000111110000010010010000 0001000000010001000100000001001100010001 0010000001011111000100010011000100010100 0001000101010000000011100000000111000001 0001011000000001100000000001001100010000 1100000000010110000100010111000100011000 000001010000

Pass2: In this pass each 12-bit block will be XOR with key, which is given in Table 3:

1111000011011111010110011110000011111111 0101101011110001110011100000111011110001 1101111100010000111100011100111100101110 1111010011111110011011111011100011111110 0100111111100100111011110101111110100000 1110111100001111111001011111111000111110 1111011011111110100011101111011111101111 1000111110110100111011111001111011111010 1111101000001111111000111111111011001110 1111101111111110100011101111000011111110 0101111111100011111011110110111111101000 1110111111001110111111011111101101001110 1111111011111010000011111100111011101111 0000111110110010111111111111111000011111 1111000110001111000100001111111100011000 1111000100001111010001101111000111111110 0001111011110001110011100001110111110101 0000111000011100111000011011111000011010 1111000000011111000100111110000110011111 0001011111110001110011100000001111110001 1001111000011000111000010111111101011111

\section{Conclusion}

A new algorithm has been proposed that focused both on the security as well as length of cipher text. The proposed algorithm produced smaller cipher text in comparison to existing methods. In this algorithm dictionaries are acting as keys and both the encoding and decoding is not possible until key is not available. The code generation is simpler in comparison to existing algorithms which are mostly based on mixed operators or values and other mathematical operations. The transmission time of cipher text has been also reduced. Also it reduces the redundancy in data representation to decrease the storage required for that data. We extending this algorithm to our future work, we will try to provide a mechanism so that there should be no need to send secondary dictionary to receiver end.

\section{Funding Information}

The authors have no support or funding to report.

\section{Author's Contributions}

All authors equally contributed in this work.

\section{Ethics}

This article is original and contains unpublished material. The corresponding author confirms that all of the other authors have read and approved the manuscript and no ethical issues involved.

\section{References}

Diffie, W. and M. Hellman, 1976. New directions in cryptography. IEEE Trans. Inform. Theory, 22: 644-654. DOI: 10.1109/TIT.1976.1055638

Diffie, W. and M.E. Hellman, 1979. Privacy and authentication: An introduction to cryptography. Proc. IEEE, 67: 397-427. DOI: 10.1109/PROC.1979.11256

Mani, A. and A. Derick, 2010. An algorithm to reduce the size of cipher text. Global J. Comput. Sci. Technol., 10: 50-54.

McConnell, M., 2002. Information Assurance in the twenty-first century. IEEE Comput., 35: 16-19. DOI: 10.1109/MC.2002.1012425 